\title{
Efficacy of dynamic elastomeric fabric orthoses in the management of scoliosis
}

\author{
Martin Mattews \\ From 7th International Conference on Conservative Management of Spinal Deformities \\ Montreal, Canada. 20-22 May 2010
}

\section{Introduction}

The use of scoliosis correcting dynamic elastomeric fabric orthoses (DEFO) have been used in the UK for a number of years for children with neurological dysfunction or deficit. The subjects would previously have been treated with rigid or semi- rigid constructed spinal jackets, however some have been reported to be less than favourable for some of the conditions. This paper will demonstrate the efficacy of using this type of dynamic orthoses as a treatment over a period of years for a number of single cases.

\section{Methods}

Seven cases have been identified to provide an overview of the outcomes possible using this innovative orthotic intervention using $x$-rays and photographs to illustrate change and maintenance over time. The conditions presented include spinal injury, cerebral palsy and agenesis of the corpus callosum. The orthoses comprise of Lycra ${ }^{\circ}$ based fabric snug fitting suits with built in panels using radiographic blueprinting processes to enable bespoke design and manufacture.

\section{Results}

Radiographs provide evidence of improved alignment and reduction in Cobb angle often maintained for several years. The orthoses allowed full range of spinal movement and some correction, which it can be hypothesized, could be due to proprioceptive feedback enabling the subject to be more aware of their body's spatial awareness. Several subjects reported the sensation of balance being off-centre.

DM.Orthotics Ltd, Norwich, Norfolk, United Kingdom

Full list of author information is available at the end of the article

\section{Discussion}

The DEFO provides further evidence of the efficacy for this type of treatment with longitudinal use suggesting longer term outcomes can be expected. Correction of the spine appears to be continuous with the natural elasticity of the fabric and designed pressure and void areas combining to enable lateral and de-rotative shifting to occur. Muscle tone also appears to improve resulting in children with cerebral palsy often able to wean out of the orthosis in time. Conventional spinal orthoses have been known to reduce muscle tone in this patient group due to muscle inactivity causing the scoliosis to progress. This suggests that conventional spinal bracing is treating the result rather than the cause of the curve. DEFOs appear to treat the unbalanced muscle tone thereby causing an effect on the resultant muscle forces impacting on the scoliosis progression.

\section{Conclusion}

The fact that the change has been initiated and controlled by DEFO scoliosis management indicates that this intervention can have positive outcomes and provides an exciting insight to the future of scoliosis orthotic management. The evidence emerging, suggests that these orthoses may become the preferred orthotic intervention for children with neurological dysfunction and deficit. Early intervention is paramount to obtain a successful outcome.

Published: 10 September 2010

doi:10.1186/1748-7161-5-S1-O51

Cite this article as: Mattews: Efficacy of dynamic elastomeric fabric orthoses in the management of scoliosis. Scoliosis 2010 5(Suppl 1):O51. 